\title{
Competitive interactions in Drosophila melanogaster: genetic variation for interference through media conditioning
}

\author{
Mortaza Hemmat and \\ Paul Eggleston
}

\author{
Department of Genetics, University of Liverpool, \\ P.O. Box 147, Liverpool. L69 3BX.
}

\begin{abstract}
Interference, which is one of two aspects of the process of competition which take place in genetically heterogeneous mixtures has been studied in the Texas population of Drosophila melanogaster. Both survival and mean adult weight were investigated in the population itself (which displays high levels of aggression and little response) and in LA, a genotype derived from the population (which displays low aggression and high levels of response) in both homotypically and heterotypically conditioned media. The results presented here show that the competitive effects of conditioning depend not only on the concentration of the conditioned medium but also on the genotype of the larvae which conditioned the medium and that of the flies which respond to such media. It was also concluded that medium conditioning is one of a range of biological parameters involved in the determination of the aggression and response components of the competitive interaction among Drosophila larvae. Thus the competitive fitness of a genotype of $D$. melanogaster is related not only to genetic variation for aggression and response but also to genetic variation in the ability to condition media and the sensitivity to such media.
\end{abstract}

\section{INTRODUCTION}

Competition is generally acknowledged to be an important selective agent in both natural and commercial populations where individuals exist in genetically heterogeneous mixtures. Despite this importance there is little understanding of the biological parameters which might determine the outcome of competition. Considerable advances have been made in the analysis of competitive interactions (Mather and Caligari, 1981; Eggleston, 1985; Spitters, 1986) and these studies have served to emphasise the genetical complexity of competition. In Drosophila melanogaster, for example, analyses of competition can now identify and estimate specific components of the competitive interaction which have been defined as aggression and response (Mather and Caligari, 1983). However, such parameters are necessarily relative in that two strong or two weak competitors might well lead to the same outcome when observed in mixture. There have been few attempts to identify (in any organism) the basic biological properties which must determine such relative components. A recent investigation of the relationship between competitive parameters (aggression and response) and bio- logical parameters (larval feeding rate and larvaadult conversion efficiency) in $D$. melanogaster emphasised the complexity of the situation (de Miranda and Eggleston, 1988 $a, b$ ). Although biological parameters such as these are clearly important they do not adequately describe the competitive interaction. Here we describe experiments which reveal genetic variation in the interference of competing larvae of $D$. melanogaster. This interference is mediated by conditioning of the growth medium with secreted or excreted metabolic agents and clearly forms part of the spectrum of biological parameters which determine the outcome of competitive encounters.

Interference is one of two aspects of the process of competition which have been defined by Birch (1957) and Bakker (1961, 1969). The other component is exploitation and this refers to the acquisition of some necessary and limited resource, for example food or light, by the competing individuals. Exploitation has long been a subject of investigation in D. melanogaster (Bakker, 1961; Mather and Caligari, 1981; de Miranda and Eggleston, 1987) and also in other organisms, for example Lolium perenne (Mather, Hill and Caligari, 1982). Interference has also been 
investigated in Drosophila although usually in the context of the competition between different species. These experiments provide evidence which suggests that larval metabolic products might be responsible (in part) for determining the viability of individuals of the same or different species (Sang, 1949; Budnik and Brncic, 1974). Weisbrot (1966) concluded that such medium conditioning, whether by the same or different genotypes, could result either in an improvement or a reduction in viability. This situation is therefore analogous to that of exploitation and facilitation with respect to the acquisition of a limited resource.

In an earlier investigation (Hemmat and Eggleston, 1988) two lines were derived from the Texas population of $D$. melanogaster by recurrent selection against a uniform inbred tester genotype. One line had been selected for low levels of aggression (LA) and the other for high response or increased sensitivity to the effects of competition (HR). The Texas population itself normally displays very high levels of aggression and low levels of response as a result of natural selection under the intensely competitive cage conditions. The LA and HR selected lines therefore display very different competitive characteristics to the Texas population. Here we describe experiments which compare the interference of the selected line (LA) and the Texas population (TP). This interference is mediated by medium conditioning which may be defined as either homotypic (self conditioning by individuals of the same species) or heterotypic (conditioned by individuals of another species). Examples of these phenomena can be found in Allee et al., (1949). In the present investigation the concept of homotypic and heterotypic conditioning is extended to include differences between genotypes within the species $D$. melanogaster.

\section{MATERIALS AND METHODS}

The two genotypes of $D$. melanogaster employed in this experiment were the Texas population (TP) and a line derived by recurrent selection from the population denoted LA (low aggression) by Hemmat and Eggleston (1988). Previous experience has shown that the population can be considered as a "genotype" for the purpose of analysis, despite its genetical heterogeneity (Eggleston, 1985).

Cultures were raised in $2.5 \times 7.5 \mathrm{~cm}$ glass vials, each containing $5 \mathrm{ml} 2$ per cent Bacto Agar to which $15 \mathrm{ml} / 1$ of 10 per cent nipagin in ethanol was added as an anti-fungal agent. In order to prepare the conditioned medium $0.45,0.90$ and $1.35 \mathrm{ml}$ of live yeast suspension were dispensed into vials for cultures with egg densitites of 60,120 and 180 eggs per vial respectively. The original concentration of this suspension was $38.4 \mathrm{~g}$ of dried bakers yeast (Sigma YSC-2) in $90 \mathrm{ml}$ distilled water. Thus each vial had a fixed quantity of $3 \cdot 2 \mathrm{mg}$ of live yeast available as food for each newly hatched larva. This quantity of yeast is slightly in excess of that shown to be necessary for each larva to successfully reach the adult stage (Bakker, 1961). In this way competition for food was eliminated or at least minimised among the developing larvae. The vials were dried overnight at $25^{\circ} \mathrm{C}$ and seeded with eggs which had been laid on plastic partitions coated in charcoal blackened starch paste. Oviposition took place in yeasted vials over a period of two hours at $25^{\circ} \mathrm{C}$ to minimise developmental heterogeneity. All competition vials were started with a counted number of eggs placed onto the surface of the food source with a sterilized needle. Densities of 60,120 and 180 eggs per vial were employed and thus for each of the two genotypes three concentrations of the following types of conditioned media were involved:

(a) Homotypically conditioned (which refers to the self-conditioning of the medium by larvae of any particular genotype).

(b) Heterotypically conditioned (which refers to the conditioning of the medium by larvae of a different genotype).

The larvae were allowed to develop at $25^{\circ} \mathrm{C}$ for 4 days. After this period they were subjected to a temperature of $-20^{\circ} \mathrm{C}$ which effectively kills the larvae. After thawing at $25^{\circ} \mathrm{C}$ these vials were considered to be "conditioned" according to the technique of Weisbrot (1966). All vials subsequently received $0.45 \mathrm{ml}$ of fresh yeast solution with the same concentration as used before to prepare the conditioned tubes for re-seeding with 60 eggs of the appropriate genotype ( $3 \cdot 2 \mathrm{mg}$ yeast per larva). The experimental design therefore consisted of four concentrations of conditioned medium $(0,60$, 120 and 180 eggs per vial) with the zero concentration represented by vials which had not been seeded with eggs but which had been frozen and thawed with the other vials. 60 eggs of the two experimental genotypes were then seeded into these vials and therefore the conditioned medium could be considered as either homotypically or heterotypically conditioned. Four replicates of each combination of genotype and concentration were raised in order to provide an estimate of error variation and all vials within each replicate were individually randomised and incubated at $25^{\circ} \mathrm{C}$. 
As the adults emerged they were counted and weighed every day in order to estimate the proportion of seeded eggs surviving to adulthood $(P)$ and the mean weight of the emerging adults $(\bar{w})$. Each value of $P$ was then converted into an angle $(\mathrm{Pa})$ using the standard angular transformation (Fisher and Yates, 1963).

The whole experiment was also carried out simultaneously in third pint milk bottles rather than vials, in order to investigate the effect of space on the observed interference. The same method was used but the amount of agar added to each bottle was $25 \mathrm{ml}$ and the experiment was duplicated rather than quadruplicated.

To determine whether the interactions between specific genotypes and conditioned media were controlled by secreted or excreted metabolic agents and not by other experimental artefacts the following comparisons (first described by Weisbrot, 1966), were made. The survival ( $P a)$ and mean weight $(\bar{w})$ of the flies emerging from the 60 eggs of each genotype seeded were compared in five different media:

(a) Control: standard competition vial containing agar and nipagin, seeded with live yeast suspension.

(b) OF: (Old and frozen) standard vial as in (a) but aged for four days at $25^{\circ} \mathrm{C}$ and then frozen at $-20^{\circ} \mathrm{C}$ to simulate the technique for killing larvae.

(c) ST: (Stirred) old and frozen vial as in (b) above but stirred to simulate the breaking up of the medium by larval activity.

(d) DP: (Dead of Population) Old and frozen vial as in (b) above but seeded with 60 dead larvae from the population.

(e) DLA: (Dead of LA) Old and frozen as in (b) and (d) above but seeded with 60 dead larvae from the LA genotype.

The last two media were employed to demonstrate that any observed interference is caused by the active larvae and therefore their metabolic products rather than by their mere presence.

\section{RESULTS}

The results obtained throughout the interference experiment are depicted in figs $1(P a)$ and $2(\bar{w})$. With respect to the character $P a$ the highest survival is always found in the control media. Under these conditions survival is higher for the Texas population (fig. 1(a), (c)) than for the genotype LA (fig. 1(b), (d)) in both tubes and bottles. Emergence of the LA genotype continues to drop as the concentration of conditioned medium increases (fig. 1(b)). There is not, however, any significant difference in the survival of the Texas population between the control value and media conditioned either homotypically or heterotypically at a density of 60 eggs per vial (fig. 1(a)). As the concentration of the conditioned medium increases, however, the survival of population individuals declines but the effect of homotypic and heterotypic conditioning is equivalent. The genotype LA does show a differing response to homotypically and heterotypically conditioned media. Survival of the LA genotype is lower when the medium is conditioned by population individuals than when it is homotypically conditioned and this effect is seen at all concentrations (fig. 1(b)). The reduced survival of the LA genotype in media conditioned by population individuals is, however, less pronounced when the experiment is raised in bottles (fig. $1(\mathrm{~d})$ ). Clearly, whatever the mechanism of interference the extra space provided in bottles produces either an environmental refuge or a dilution effect which reduces the effect of the conditioning. There is no evidence that the population individuals are similarly affected by the extra space available in bottles.

With respect to the character $(\bar{w})$, the highest mean adult weight is achieved by individuals from the Texas population in non conditioned (control) media (fig. 2a)). As the concentration of conditioned medium increases, mean adult weight falls and this trend is more pronounced when the medium is homotypically conditiond. Conditioning heterotypically by the LA genotype is less efficient at reducing the mean adult weight of the population flies. The effect of conditioning on flies of the LA genotype is much more dramatic (fig. 2(b)). Mean adult weight falls sharply even at the lowest concentration of conditioned medium used. There is little further change as concentration increases and this probably reflects the critical weight (minimum pupation weight for survival) of the LA genotype. Interestingly, there is no difference in the efficacy of the two types of conditioned medium for the LA genotypt. As with the character $(P a)$ all effects observed in bottles (fig. $2(c),(d))$ are less pronounced and the effects of homotypic and heterotypic conditioning are equivalent. This probably reflects the dilution of conditioning metabolites in the larger bottle volume.

The results depicted in figs 1 and 2 were subjected to a factorial analysis of variance (table 1). For both characters, the variation between indicator genotypes, between concentrations of conditioned 

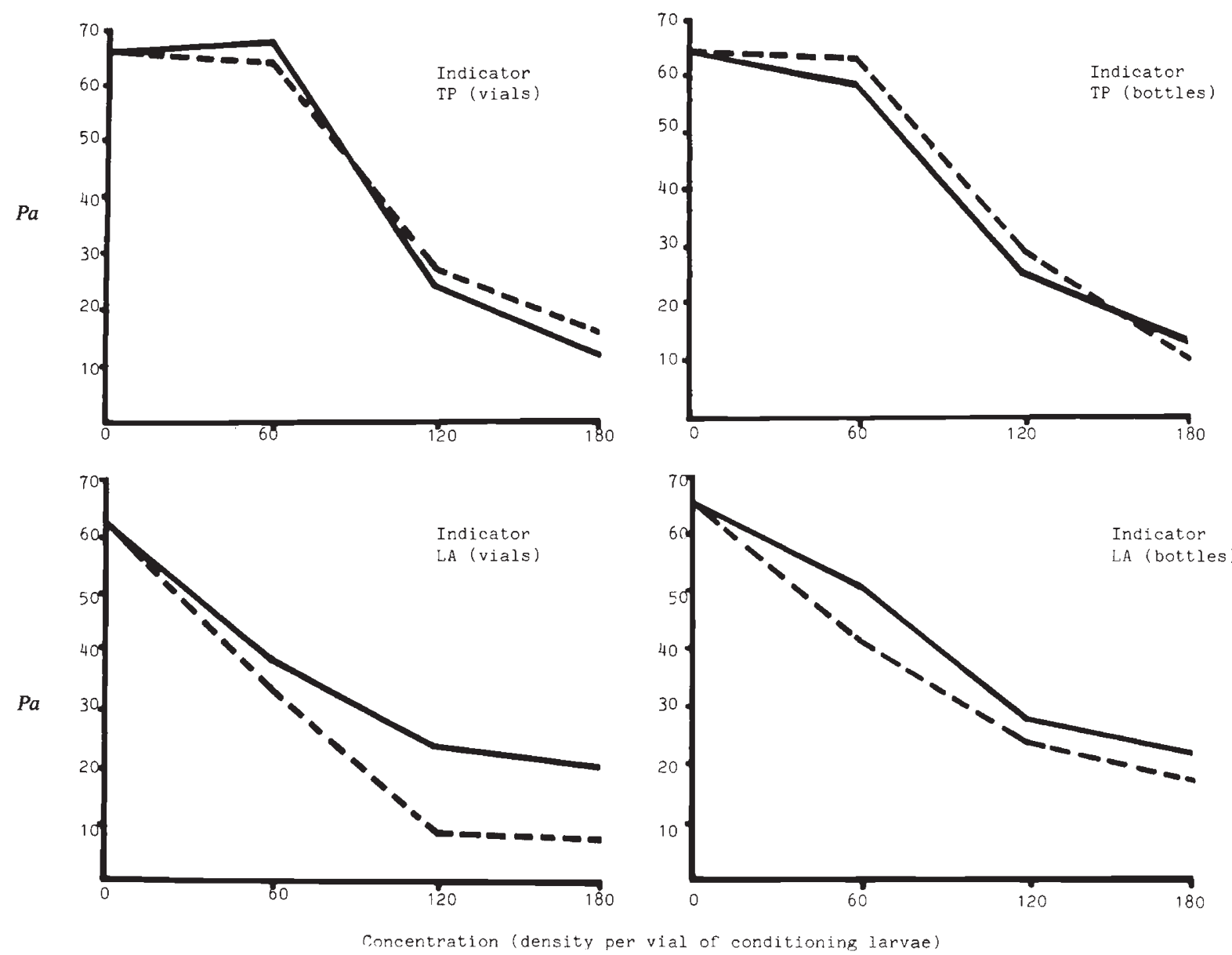

Figure 1 The effects of medium conditioning on the survival ( $P a$ ) of two strains of Drosophila melanogaster, namely Texas population (TP) and low aggression (LA). In each graph the points represent the average proportion of seeded eggs of the indicator genotype surviving to adulthood following medium conditioning by varying concentrations of either (TP) larvae (broken lines) or (LA) larvae (solid lines). The experiment was carried out in both vials and bottles.

media and between conditioning genotypes were found to be significant in all but one case. As expected, the levels of significance of each main effect reflect the more pronounced differences observed in vials as opposed to bottles and the one non-significant main effect concerns the effect of conditioning genotypes on mean weight of adults emerging in bottles. The various interaction items in the analysis of variance are also quite informative. In all cases the largest interaction item is that which reflects the inconsistent response of the two indicator genotypes to different concentrations of conditioned medium $(\mathrm{A} \times \mathrm{B})$. The significant interaction is largely attributable to the different response of the two indicator genotypes at the lowest $(60)$ concentration of conditioned medium. In general, population individuals have a much less pronounced response to medium conditioning, particularly at low concentrations. This was not unexpected since the two indicator genotypes were chosen because of their divergent behaviour. Only in one case (mean weight of flies raised in bottles) was this item not significant. However, the analysis of variance shows that rather little variation was found in general in this latter case; only differences between indicator genotypes and concentrations being significant. The same exception applies to the next most significant interaction (indicator genotype $\times$ conditioning genotype; $[\mathrm{A} \times \mathrm{C}]$ ) which again emphasises the divergent behaviour of the chosen indicator genotypes. For the character $(P a)$, population individuals tend to be equally affected by homotypic and heterotypic conditioning whereas the genotype LA shows a differential 

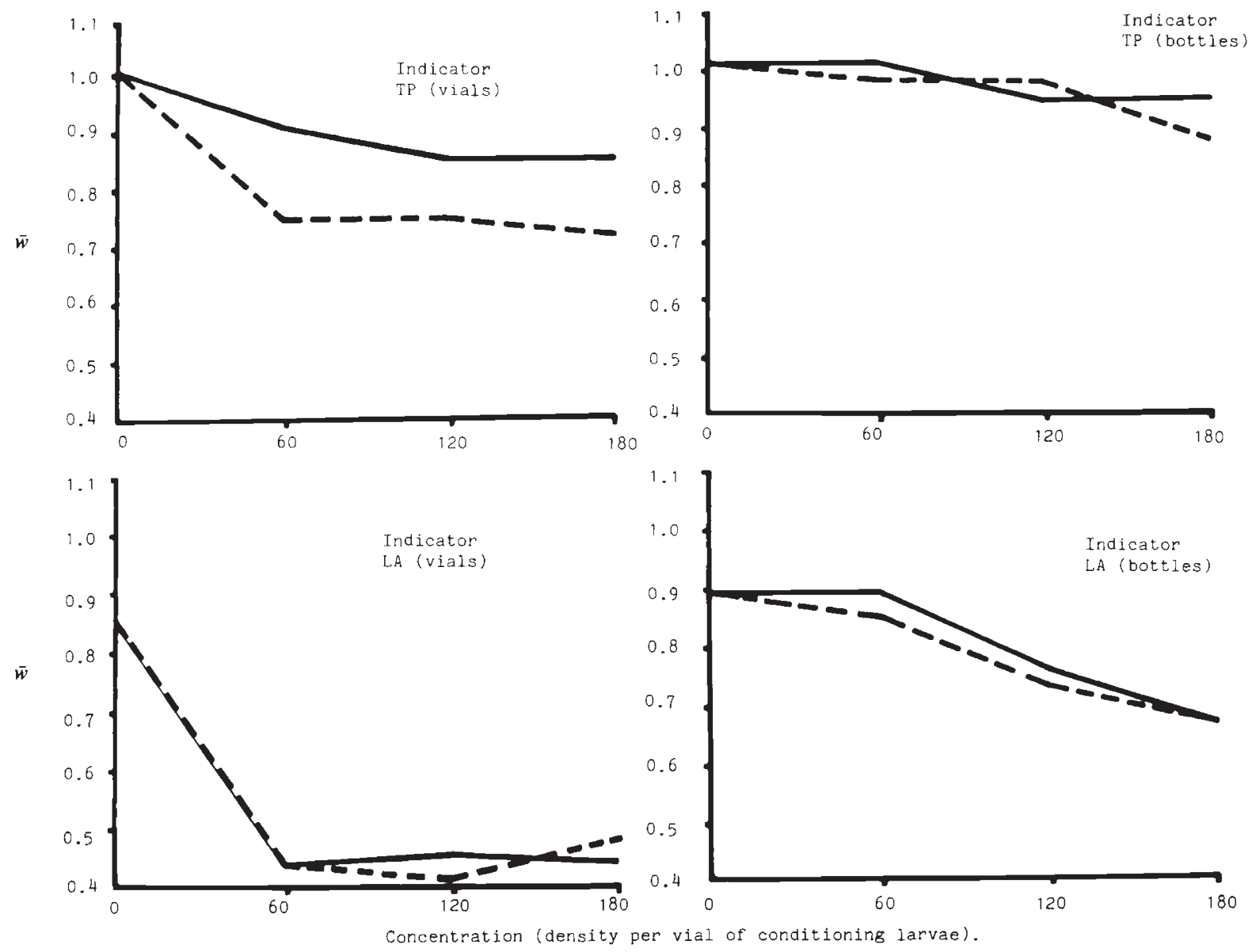

Figure 2 The effects of medium conditioning on the mean weight of surviving adults ( $\bar{w})$ for the Texas population (TP) and low aggression (LA) strains of Drosophila melanogaster. Mean weights (mg) for the indicator genotype are given following medium conditioning by varying concentrations of either (TP) larvae (broken lines) or (LA) larvae (solid lines). The experiment was carried out in both vials and bottles.

response with survival reduced more markedly by heterotypic conditioning (fig. 1(a), (b)). This situation is reversed for the character $(\bar{w})$ where the population individuals show a differential response with their mean adult weight being reduced more effectively by homotypic medium conditioning (fig. 2(a), (b)). These trends are not evident when the experiment is raised in bottles. Differences between the conditioning genotypes tend to be consistent at all concentrations as shown by the universally insignificant interaction item $(\mathrm{B} \times \mathrm{C})$. These interactions are reflected much as would be expected in the three way interaction item $(\mathrm{A} \times \mathrm{B} \times \mathrm{C})$ which is generally rather small although significant in three out of four cases (table 1).

An additional experiment was carried out to determine if the decrease in viability and decline in weight of the two indicator genotypes in the conditioned media were caused by active larvae and their metabolic products and not by other extraneous factors or artefacts. In this respect, the survival $(P a)$ and mean adult weight $(\bar{w})$ of the flies resulting from 60 eggs of each indicator genotype seeded onto control (non conditioned) medium were compared with comparable measurements taken on four other media. It can be seen from the analysis of variance of the results (table 2) that overall there are no significant differences in survival or mean adult weight between the five different media and that there is no evidence of any overall interaction between genotypes and media. In order to determine whether or not any particular differences might be significant the variation among the media totals (summed over both genotypes) can be partitioned. 
Table 1 Analysis of variance of the experimental results for survival and mean weight of surviving adults which are depicted in figs 1 and 2

\begin{tabular}{|c|c|c|c|c|c|c|}
\hline \multirow[b]{2}{*}{ Source of variation } & \multicolumn{3}{|l|}{ Vials } & \multicolumn{3}{|l|}{ Bottles } \\
\hline & $\begin{array}{l}\text { Degrees of } \\
\text { freedom }\end{array}$ & $\begin{array}{l}\text { Survival } \\
\text { (mean squares) }\end{array}$ & $\begin{array}{l}\text { Mean weight } \\
\text { (mean squares) }\end{array}$ & $\begin{array}{l}\text { Degrees } \\
\text { of freedom }\end{array}$ & $\begin{array}{l}\text { Survival } \\
\text { (mean squares) }\end{array}$ & $\begin{array}{l}\text { Mean weight } \\
\text { (mean squares) }\end{array}$ \\
\hline (A) Indicator genotype & 1 & $1870 \cdot 67^{* * *}$ & $1 \cdot 5750^{* * *}$ & 1 & $20 \cdot 18^{*}$ & $0 \cdot 2965^{* * *}$ \\
\hline (B) Concentration & 3 & $9323 \cdot 53^{* * *}$ & $0.4091^{* * *}$ & 3 & $4078 \cdot 16^{* * *}$ & $0 \cdot 0540^{* * *}$ \\
\hline (C) Conditioning genotype & 1 & $206 \cdot 03 * * *$ & $0.0473^{* * *}$ & 1 & $21 \cdot 50^{*}$ & $0.0012 \mathrm{~ns}$ \\
\hline $\mathrm{A} \times \mathrm{C}$ & 1 & $294 \cdot 16^{* * *}$ & $0.0297^{* * *}$ & 1 & $85 \cdot 57^{* * *}$ & $0.0000 \mathrm{~ns}$ \\
\hline $\mathrm{A} \times \mathrm{B} \times \mathrm{C}$ & 3 & $81 \cdot 56^{*}$ & $0.0063^{*}$ & 3 & $19 \cdot 98^{* * *}$ & $0.0010 \mathrm{~ns}$ \\
\hline Error & 48 & $25 \cdot 15$ & 0.0022 & 16 & $4 \cdot 26$ & 0.0015 \\
\hline Total & 63 & & & 31 & & \\
\hline
\end{tabular}

Levels of significance are given as ns, $P>0.05 ;^{*}, 0.05>P>0.01 ; * *, 0.01>P>0.001$ and $* * *, P<0.001$. See text for details.

Table 2 Analysis of variance of the survival and mean weight of indicator genotype individuals when raised on five different media. Levels of significance are as given in table 1 . The comparisons $\mathrm{C} 1$ to $\mathrm{C} 4$ reflect an orthogonal partition of the variation among media totals and the comparisons $\mathrm{C1}^{\prime}$ to $\mathrm{C}^{\prime}$ ' reflect a similar partition of the medium $\times$ genotype interaction item. See table 3 and text for details

\begin{tabular}{|c|c|c|c|}
\hline $\begin{array}{l}\text { Source of } \\
\text { variation }\end{array}$ & $\begin{array}{l}\text { Degrees } \\
\text { of freedom }\end{array}$ & $\begin{array}{l}\text { Survival ( } P a \text { ) } \\
\text { (mean squares) }\end{array}$ & $\begin{array}{l}\text { Mean weight }(\bar{w}) \\
\text { (mean squares) }\end{array}$ \\
\hline (A) Indicator genotype & 1 & $373 \cdot 38210^{* * *}$ & $0.336722^{* * *}$ \\
\hline (B) Medium & 4 & $24 \cdot 65849 \mathrm{~ns}$ & $0.001022 \mathrm{~ns}$ \\
\hline C1 Treatment versus control & 1 & $4 \cdot 63080 \mathrm{~ns}$ & $0.000640 \mathrm{~ns}$ \\
\hline C2 Stirring & 1 & $17 \cdot 20176 \mathrm{~ns}$ & $0.000100 \mathrm{~ns}$ \\
\hline C3 Addition of dead larvae & 1 & $69 \cdot 03125 \mathrm{~ns}$ & $0.002450 \mathrm{~ns}$ \\
\hline C4 DP versus DLA larvae & 1 & $7 \cdot 77016 \mathrm{~ns}$ & $0.000900 \mathrm{~ns}$ \\
\hline $\mathrm{A} \times \mathrm{B}$ & 4 & $7 \cdot 34067 \mathrm{~ns}$ & $0.000625 \mathrm{~ns}$ \\
\hline $\mathrm{C} 1^{\prime}=\mathrm{C} 1 \times \mathrm{A}$ & 1 & $0 \cdot 15252 \mathrm{~ns}$ & $0.000302 \mathrm{~ns}$ \\
\hline $\mathrm{C} 2^{\prime}=\mathrm{C} 2 \times \mathrm{A}$ & 1 & $2 \cdot 22605 \mathrm{~ns}$ & $0.001600 \mathrm{~ns}$ \\
\hline $\mathrm{C} 3^{\prime}=\mathrm{C} 3 \times \mathrm{A}$ & 1 & $12 \cdot 97801 \mathrm{~ns}$ & $0.000612 \mathrm{~ns}$ \\
\hline $\mathrm{C} 4^{\prime}=\mathrm{C} 4 \times \mathrm{A}$ & 1 & $14 \cdot 00631 \mathrm{~ns}$ & $0.000025 \mathrm{~ns}$ \\
\hline Error & 30 & $32 \cdot 08456$ & 0.000943 \\
\hline Total & 39 & & \\
\hline
\end{tabular}

An appropriate partition providing four orthogonal comparisons is given in table 3 . These comparisons test the average effect of the treatments versus the control ( $\mathrm{C} 1)$, the effect of stirring the medium (C2), the effect of the addition of dead larvae (C3) and the effect of the difference between the two sources of dead larvae (C4). The same comparisons, based on the difference between the two genotypes (within each medium) reveal how the different media interact with the two genotypes and provide a suitable partition of the $(A \times B)$ interaction item (denoted $\mathrm{Cl}^{\prime}$ to $\mathrm{C}^{\prime}$ ). The mean squares resulting from these comparisons (each for $1 \mathrm{df}$ ) are given in table 2 and it can be seen that none of the items approach significance. In fact, the only significant item in the analysis is that which reflects the expected differences between the two indicator genotypes, namely the higher survival and mean adult weight attained by the Texas population at this egg density. This finding supports the hypothesis that some agent (or agents) in the larval metabolic wastes of the conditioning genotype are responsible for the observed decline in survival and mean adult weight of each indicator genotype with increasing concentration of conditioned medium. 
Table 3 Media totals (summed over both genotypes) and media differences (difference between two genotypes) for each of the media involved in the experiment. An appropriate partition of the variation among the media totals yields four orthogonal comparisons. The same comparisons applied to the media differences produce a partition of the Indicator genotype $\times$ Medium interaction sum of squares. See table 2 for appropriate sums of squares and text for details

\begin{tabular}{|c|c|c|c|c|c|c|}
\hline & & \multicolumn{4}{|l|}{ Medium } & \multirow[b]{2}{*}{ Control } \\
\hline & & OF & ST & DP & DLA & \\
\hline Media totals & $\begin{array}{l}P a \\
\bar{w}\end{array}$ & $\begin{array}{r}511 \cdot 36 \\
7 \cdot 44\end{array}$ & $\begin{array}{r}494 \cdot 77 \\
7 \cdot 48\end{array}$ & $\begin{array}{r}532 \cdot 14 \\
7 \cdot 54\end{array}$ & $\begin{array}{r}520.99 \\
7.66\end{array}$ & $\begin{array}{r}508 \cdot 01 \\
7 \cdot 61\end{array}$ \\
\hline Media differences & $\begin{array}{l}P a \\
\bar{w}\end{array}$ & $\begin{array}{r}-14.88 \\
-0.86\end{array}$ & $\begin{array}{r}-29 \cdot 29 \\
-0.70\end{array}$ & $\begin{array}{r}-18 \cdot 82 \\
-0.70\end{array}$ & $\begin{array}{r}-33.79 \\
-0.72\end{array}$ & $\begin{array}{r}-25.43 \\
-0.69\end{array}$ \\
\hline Comparisons & $\begin{array}{l}\mathrm{C} 1 \\
\mathrm{C} 2 \\
\mathrm{C} 3 \\
\mathrm{C} 4\end{array}$ & $\begin{array}{r}-1 \\
1 \\
-1 \\
0\end{array}$ & $\begin{array}{r}-1 \\
-1 \\
-1 \\
0\end{array}$ & $\begin{array}{r}-1 \\
0 \\
1 \\
1\end{array}$ & $\begin{array}{r}-1 \\
0 \\
1 \\
-1\end{array}$ & $\begin{array}{l}4 \\
0 \\
0 \\
0\end{array}$ \\
\hline
\end{tabular}

\section{DISCUSSION}

The experiments reported in this paper support the conclusions of previous studies in $D$. melanogaster in that conditioning of the growth medium by biotic residues can be responsible for variation in competitive ability and viability (Dawood and Strickberger, 1964, 1969; Sang, 1949; Weisbrot, 1966; and Dolan and Robertson, 1975). In addition we have also shown that the competitive effects of conditioning depend not only on the concentration of the conditioned medium but also on the genotype of the larvae which conditioned the medium and that of the flies which respond to such media. The observed differences between the two lines studied here (Texas population and selected strain LA) suggest that medium conditioning is one of a range of biological parameters involved in the determination of the aggression and response components of competitive interactions. Other such parameters, including larval feeding rates and conversion efficiencies have been reported elsewhere (de Miranda and Eggleston, 1988 $b, c)$. The Texas population, for example, has a high level of aggression and little response in competitive situations. This aggression is reflected in the ability of Texas larvae to interfere with the viability of LA individuals through medium conditioning. Their low response however, means that Texas larvae are less sensitive to medium conditioned by an associate genotype (in this case, LA).

The demonstrated genetic variation in the ability to condition media and the sensitivity to such media means that the adaptive value or competitive ability of a genotype will be influenced by the environment in which it develops. As a result it can be suggested that fitness (in terms of competition) is related to the genotype of associate or contemporary competitors. Many previous studies have also stressed the importance of the genetic dimension of the environment (Levene et al., 1954, 1958; Lewontin, 1955; Lewontin and Matsuo, 1963 and Sokal and Karten, 1964). The complexity of this genotype/environment interaction is emphasised by the differing response of the two competitive characters, $\mathrm{Pa}$ and $\bar{w}$. For example, although individuals of the Texas population were much more efficient than those of the LA genotype at conditioning the growth $\cdot$ medium and thereby interfering with the survival of genotype LA, no such difference was observed in respect of the mean weight of LA adults.

Without further knowledge of the biochemical nature of the conditioning metabolites it is impossible to say whether the genotypic differences in conditioning ability are qualitative or merely quantitative. The fact that the LA genotype was derived from the Texas population by recurrent selection favours the latter alternative although qualitative differences cannot be ruled out. It is now clear that any attempt to describe the biological nature of competitive ability (even the relatively simple "scramble" type of competition among Drosophila larvae) must take into account a wide and complex range of parameters including not only feeding rates, conversion efficiencies and biotic residues of the competing larvae themselves but also the nature of the conditioning imposed by contemporary competitors. 


\section{REFERENCES}

ALleE, W. C., EMERSON, A. E., PARK, O., PARK, T. AND SCHMIDT, K. P. 1949. Principles of Animal Ecology. W. B. Saunders Co., Philadelphia, 837 pp.

BAKKER, K. 1961. An analysis of factors which determine success in competition for food among larvae of Drosophila melanogaster. Arch. Netherland. Zool, 14, 200 281.

BAKKER, K. 1969. Selection for rate of growth and its influence in competitive ability of larvae of Drosophila melanogaster. Netherlands Journal of Zoology, 19, 541-595.

BIRCH, L. C. 1957. The meaning of competition. The American Naturalist. XCI (91), 5-18.

BUDNIK, M. AND BRNCIC, D. 1974. Pre-adult competition between Drosophila pavani and Drosophila melanogaster, Drosophila simulans, and Drosophila willistoni. Ecology, 55, 657-661.

DAWOOD, M. AND STRICKBERGER, M. 1964. The effect of larval interaction on viability in Drosophila melanogaster, I. Changes in heterozygosity. Genetics, 50, 999-1007.

DAWOOD, M. AND STRICKBERGER, M. 1969. The effect of larval interaction on viability in Drosophila melanogaster, III. Effect of biotic residues. Genetics, 63, 213-220.

de MIRANDA, J. R. AND EGGLESTON, P. 1987. A comparison of substitution and addition designs for the analysis of competitive interactions in Drosophila melanogster. Heredity, 58, 279-288.

de MIRANDA, J. R. AND EGGLESTON, P. 1988a. Larval competition in Drosophila melanogaster I. Estimation of larval growth parameters. Heredity, 60, 205-212.

de MIRANDA, J. R. AND EGGLESTON, P. 1988b. Larval competition in Drosophila melanogaster II. Comparing biological and competitive parameters. Heredity, 60, 213-219.

DOLAN, R. AND ROBERTSON, A. 1975. The effect of conditioning the medium in Drosophila, in relation to frequency dependent selection. Heredity, 35, 311-316.

EGGLESTON, P. 1985. Variation for aggression and response in the competitive interaction of Drosophila melanogaster. Heredity, 54, 43-51.
FISHER, R. A. AND YATES, F. 1963. Statistical Tables for Biological, Agricultural and Medical Research, 6th Edn. Oliver and Boyd, Edinburgh.

HEMMAT, M. AND EGGLESTON, P. 1988. Competitive interactions in Drosophila melanogaster: recurrent selection for aggression and response. Heredity, 60, 129-137.

LEVENE, H., PAVLOVSKY, O. AND DOBZHANSKY, T. 1954. Interactions of the adaptive values in polymorphic experimental population of Drosophila pseudoobscura. Evolution, 8, 335-349.

LEVENE, H., PAVLOVSKY, O. AND DOBZHANSKY, T. 1958. Differences in the adaptive values of certain genotypes in Drosophila pseudoobscura on the composition of the gene pool. Evolution, 12, 18-23.

LEWONTIN, R.C. 1955. The effects of population density and composition on viability of Drosophila melanogaster. Evolution, 9, 27-41.

LEWONTIN, R. C. AND MATSUO. 1963 Interaction of genotypes determining viability in Drosophila busckii. Proc. Nat. Acad. Sci., 49, 270-278.

MATHER, K. AND CALIGARI, P. D. S. 1981. Competitive interactions in Drosophila melangoaster, II. Measurement of competition. Heredity, 46, 239-254.

MATHER, K., HILL, J. AND CALIGARI, P. D. S. 1982. Analysis of competitive ability among genotypes of perennial ryegrass. Heredity, 48, 421-434.

MATHER, K. AND CAligari, P. D. S. 1983. Pressure and response in competitive interactions. Heredity, 51, 435-454.

SANG, J. H. 1949. The ecological determinants of population growth in a Drosophila culture. III. Larval and pupal survival. Physiol. Zool., 22, 183-202.

SOKAL, R. R. AND KARTEN, I. 1964. Competition among genotypes in Tribolium castaneum at varying densities and gene frequencies (the black locus). Genetics, 49, 195-210.

SPITTERS, C. J. T. 1986. A confrontation between a descriptive and an explanatory approach to inter-genotypic competition. Proc. 6th Meeting EUCARPIA Section. Biometrics in Plant Breeding, pp. 205-229.

WEISBROT, D. R. 1966. Genotypic interactions among competing strains of Drosophila. Genetics, 53, 427-435. 